\title{
Mathematical Modelling of the Synthesis of New Materials
}

\author{
Valery Antonov ${ }^{1}$, Roman Davidov ${ }^{1}$, Nikolay Kalinin ${ }^{2}$ and Alexey Ryakhovskiy ${ }^{2 *}$ \\ ${ }^{1}$ Peter the Great Saint-Petersburg Polytechnical University, 195251 Saint-Petersburg, Russia \\ ${ }^{2}$ Ioffe Physical-Technical Institute of Russian Academy of Sciences, 194021 Saint-Petersburg, Russia
}

\begin{abstract}
The paper focuses primarily on a magnetohydrodynamical approach to solving the numerical problems arising in studying the high energy input technologies of the synthesis of new materials. The underlining mathematical model combines the description of both electromagnetic and hydrodynamic phenomena for the metals and surrounding media in supercritical conditions. A detailed specification of this model is provided. The crucial role of the correct choice of equation of state is outlined. The results section includes the latest finding regarding the numerical simulation of underwater electrical explosion.
\end{abstract}

\section{Introduction}

Powerful electrical discharges in the plasma channels produced in liquids are widely used in scientific research and development of modern advanced technologies. A large part of applied research is directed towards the use of hydrodynamic pulses for the treatment of materials. High efficiency energy conversion, introduced into the plasma, to the fluid motion, provides a variety of technological applications of electrical discharges [1].

In this regard, a series of simplified discharge models useful for solving a given problem have been developed [2]. Some of the most accuratemathematical description of the discharge areradiative magnetohydrodynamics (RMHD) models [3]. These models allow analyzing the change in the physical condition of plasma and liquid based on the current pulses, generated by the discharge circuit.

It is equally important to determine the role of the chemical reactions as effective method for phase formation in polycrystalline systems. This approach can create models adequately describing the process of changing the state of matter as a result of the high energy supply in a short period of time.

Our scientific interest towards the powerful electrical discharge in the water is caused by the aim of getting supercritical water conditions under which it acquires properties of effective solvent. The study of the reaction gives the possibility to get physical-chemical condition of fluid required for the implementation in various technological processes [4]. 


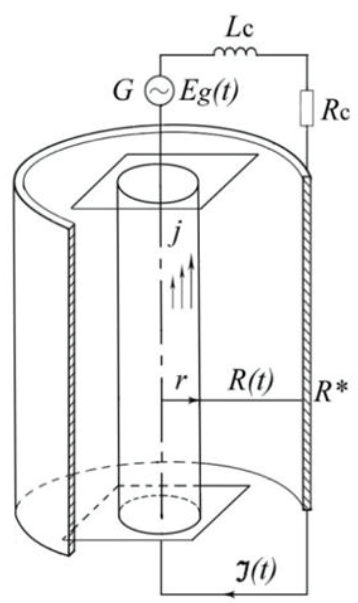

Fig. 1.Scheme of an electrical explosion setup.

Discharge power supply system consists of a high-voltage pulse generator $E g(t)$, exacerbating fuses, inductor $L_{c}$ and an electric circuit of the transmission line, which is represented in the scheme by a wave resistance $R_{c}$. The plasma channel can be regarded as a conductor, having a variable radial conductivity and inductance, and its capacity can be neglected. Pulse load - high-current discharge - represented in the diagram variable as an impedance plasma channel $R(t)$ and the inductance of the area enclosed between the plasma channel and the reverse current lead $L_{d}(t)$.

\section{Mathematical model}

The aim of this study is developing a mathematical model of the sharp changes in the phase state of a substance. To construct a mathematical model, a phenomenological method of thermal dynamics, RMHD and statistical physics has been created [5]. This approach allows developing a model which can efficiently describe the processes of heat and mass transfer during the inelastic deformation of materials.

An important step in the work is an application of the aformentioned models to describe the phase formation.

Electrical breakdown of the discharge gap initiates the discharge, forming a plasma channel in water. The phenomenon of electrical breakdown of water is not considered. The radius of the channel and the resulting plasma properties are treated as parameters of the problem and must be specified.

The plasma temperature in the discharge channel can reach tens of thousands of degrees, and heat radiation in the energy balance is beginning to play a significant role. To calculate the characteristics of the radiation in the continuous spectrum used a simplified model based on the approach of "forward - backward" (Schuster-Schwarzschild approximation) with only two spectral bands [6].

\subsection{Basic equations}

The basic set of MHD equations is as follows [5]:

$$
\frac{\partial}{\partial t} \rho+\frac{1}{r} \frac{\partial}{\partial r}(r \rho u)=0
$$




$$
\begin{gathered}
\left.\frac{\partial}{\partial t} \rho u\right)+\frac{1}{r} \frac{\partial}{\partial r}\left[r\left(\rho u^{2}+\tau\right)\right]+\frac{\partial}{\partial r}\left(P+P_{\omega}\right)+j B=0 \\
\frac{\partial}{\partial t} W+\frac{1}{r} \frac{\partial}{\partial r}\left[r u\left(\varepsilon+P+P_{\omega}+\tau\right)+r \frac{\partial T}{\partial r}\right]=\sigma E^{2}-Q_{R} \\
\frac{1}{r} \frac{\partial}{\partial r}(r B)=\mu_{0} j ; j=\sigma E \\
W=\rho\left(\varepsilon+\frac{1}{2} u^{2}\right) ; \tau=\left(\frac{3}{4} \frac{\partial u}{\partial r}-\frac{2}{3} \frac{u}{r}\right) \\
P=P(T, \rho), \varepsilon=\varepsilon(T, \rho)
\end{gathered}
$$

\subsection{Electrical circuit equation}

$$
\begin{gathered}
U_{G}(t)=L_{0} \frac{d I}{d t}+R_{0} I+\frac{d}{d t}\left(L_{D} I\right)+R_{\rho} I+E_{s} \\
R_{D}(t)=I(t)^{-2} \int_{0}^{r_{p l}(t)} \sigma E^{2} r d r
\end{gathered}
$$

$U_{G}(t)$ - strength of the electric field; $L_{0}, R_{0^{-}}$inductance and resistance in an electrical circuit; $R \rho$-impedance transporting line; $L_{D}$ - inductance of discharge plasma- reverse conductor of electric current; $E_{s}$-electrical field strength on the border out of the discharge.

\subsection{Lagrangian mass variables}

$$
s=\int_{x_{0}}^{x} \rho(y, t) d y
$$

The system of equations of gas dynamics for a one-dimensional plane nonstationary flow in Lagrangian mass coordinates takes the form

$$
\begin{gathered}
\frac{\partial}{\partial t}\left(\frac{1}{\rho}\right)=\frac{\partial v}{\partial s} ; \frac{\partial v}{\partial t}=\frac{-\partial p}{\partial s} ; \frac{\partial x}{\partial t}=v, \\
\left.\frac{\partial}{\partial t}\left(\varepsilon+\frac{v^{2}}{2}\right)=\frac{-\partial}{\partial s} p v\right)-\frac{\partial W}{\partial t} \\
p=p(\rho, T) ; \varepsilon=\varepsilon(\rho, T) .
\end{gathered}
$$

\subsection{Radiative gas}

$$
\begin{gathered}
\frac{\partial r}{\partial t}=u, \rho r d r=d m, \\
\left.\frac{\partial u}{\partial t}=-r \frac{\partial}{\partial m} p+\omega\right)+\frac{F}{\rho}, F=\frac{1}{c} j \times H, \\
\left.\frac{\partial \varepsilon}{\partial t}=-(p+\omega) \frac{\partial}{\partial t}\left(\frac{1}{\rho}\right)-\frac{\partial}{\partial m} W+W_{T}\right)+\frac{q}{\rho}, q=\frac{j_{z}^{2}}{\sigma}, \\
\frac{\partial}{\partial t}\left(\frac{H_{\varphi}}{r \rho}\right)=\frac{\partial E_{z}}{\partial m}, E_{z}=\frac{\rho}{4 \pi \sigma} \frac{\partial}{\partial m}\left(r H_{\varphi}\right), j_{z}=\sigma E_{z}, \\
W_{T}=-\chi \rho \frac{\partial T}{\partial m}, W=\sum_{k=1}^{n} r \int_{-1}^{1} \frac{\mu d \mu}{\sqrt{1-\mu^{2}}} \int_{-1}^{1} \sqrt{1-\gamma^{2}} I_{k} d \gamma
\end{gathered}
$$




\subsection{Resolving the shockwave}

Tocalculatetheshockwave,

additionaldissipativeterms

(artificialviscosity) areintroducedintothefinite difference equations. Instead of the wave front, its spectral representation is obtained. The equations of gas dynamics are written as follows

$$
\left.\frac{\partial}{\partial t}\left(\frac{1}{\rho}\right)=\frac{\partial v}{\partial s}, \frac{\partial v}{\partial t}=\frac{-\partial}{\partial s} p+\omega\right), \frac{\partial \varepsilon}{\partial t}=-(p+\omega) \frac{\partial v}{\partial s}, \omega=-v \rho \frac{\partial v}{\partial s}
$$

Artificial viscosity is an additive to pressure. It is calculated at half-integer grid points (at the boundary of the reference volume) [5].

\subsection{Equations of state}

An important step in modelling is to create an equation of state - a thermodynamic equation describing the state of matter. This equation provides a mathematical relationship between state functions such as its temperature, pressure, volume, or internal energy.

The most promising for this class of problems is to use the free energy of the system, taking into account various interaction factorsbetween the particles. It is also possible to use simpler equations of state for the internal energy of the system.

Table 1. Thermodynamic variables

\begin{tabular}{|c|c|c|c|}
\hline Name & Symbol & Formula & Natural variables \\
\hline Internal energy & $U$ & $\left.\int(T d S-p d V)+\sum_{i} \mu_{i} d N_{i}\right)$ & $S, V,\left\{N_{i}\right\}$ \\
\hline $\begin{array}{c}\text { Hemholtz free } \\
\text { energy }\end{array}$ & $F$ & $U-T S$ & $T, V,\left\{N_{i}\right\}$ \\
\hline Enthalpy & $H$ & $U+p V$ & $S, p,\left\{N_{i}\right\}$ \\
\hline Gibbs free energy & $G$ & $U+p V-T S$ & $T, p,\left\{N_{i}\right\}$ \\
\hline
\end{tabular}




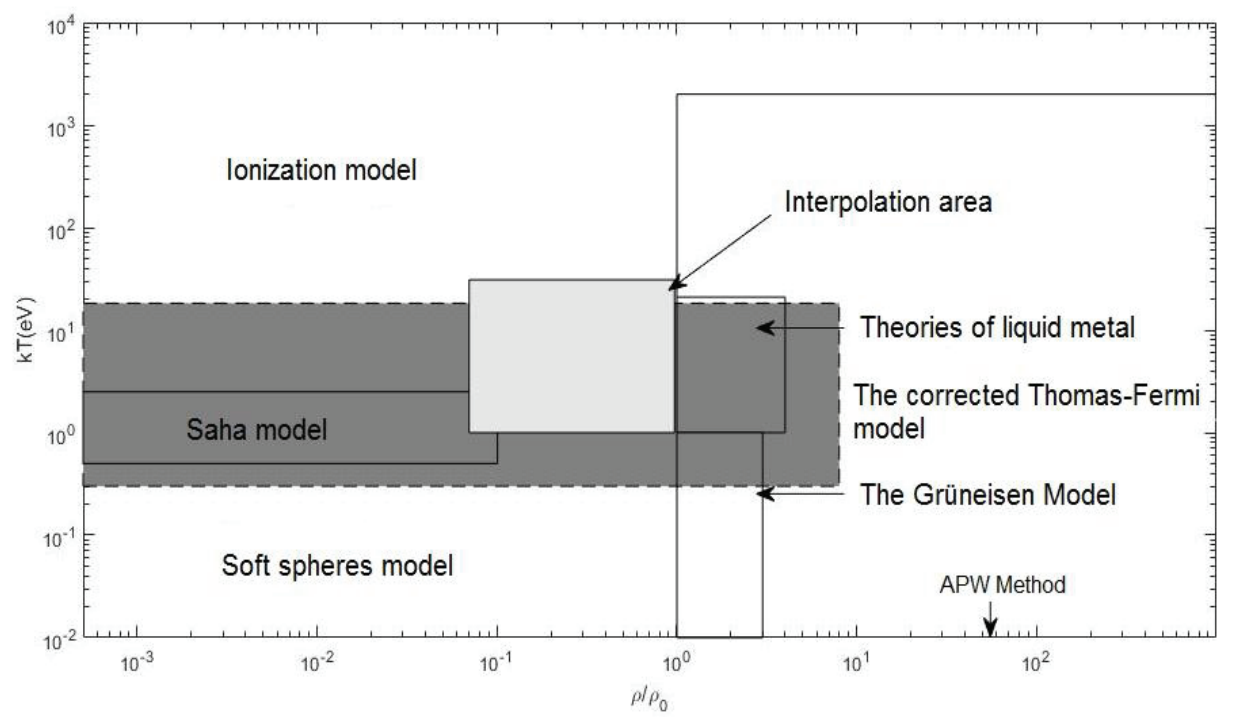

Fig. 2. Diagram of different equation of state approaches. In dark grey is an area of interest for electrical explosion of conductors.

An equation of state can be applied to either vapour-liquid or supercritical phenomena without any conceptual difficulties. Many equations of state have been proposed in the literature with either an empirical, semi-empirical or theoretical basis. Comprehensive reviews can be found in the works of Martin (1979), Gubbins (1983), Anderko (1990), Sandler (1994), Economou and Donohue (1996), Wei and Sadus (2000) and Sengers et al. (2000).

The free Helmholtz energy $\mathrm{F}$ used the elastic part of the interaction $\left(F_{c}\right)$ [8]:

$$
F=F_{e}+F_{a}+F_{c}
$$

For example,

$$
F_{a}=\frac{3 T}{2 m_{a}} \ln \left(\frac{\theta^{2}}{T^{2}}+\frac{\alpha\left(V_{0} / V\right)^{2 / 3}}{T}\right)
$$
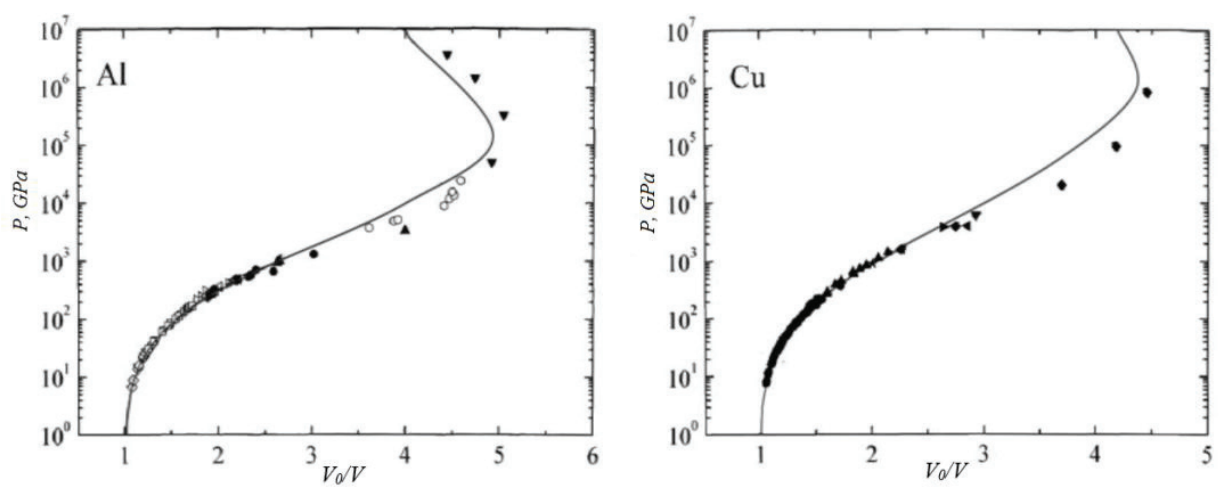

Fig. 3. Shock adiabats for $\mathrm{Al}$ and $\mathrm{Cu}$.

\subsubsection{Kolgatin equation of state}


There are restrictions for the applicability of this equation on the temperature, which should be less than $10^{5} \mathrm{~K}$ and for the pressure $\left(\mathrm{P} \leq 10^{4} \mathrm{MPa}\right)$ (includes supercritical water conditions).

For gas:the pressure is created by molecules, atoms and ions

$$
p=300[(1-\beta)+2 \beta(1+Z)] \rho T
$$

The internal energy is composed of the thermal energy of molecules and atoms, ions, electrons motion, ionization and dissipationterms

$$
\varepsilon=\varepsilon_{m}+\varepsilon_{a, i, e}+\varepsilon_{I}+\varepsilon_{d}
$$

For water:the pressure consists of elastic and thermal components:

$$
p=p_{e l}+p_{T}
$$

Internal energy

$$
\varepsilon=\varepsilon_{e l}+\varepsilon_{T}+\varepsilon_{I}+\varepsilon_{d}
$$

\section{Results}

The results below highlight the recent finding regarding the numerical simulation of underwater electrical explosions. We consider two real-life experiments with a different timescale: microsecond [9] and nanosecond [10]. On the graphs below circles indicate experimental data, cross and stars - numerical data from the original source, and point-dash line - our numerical results.

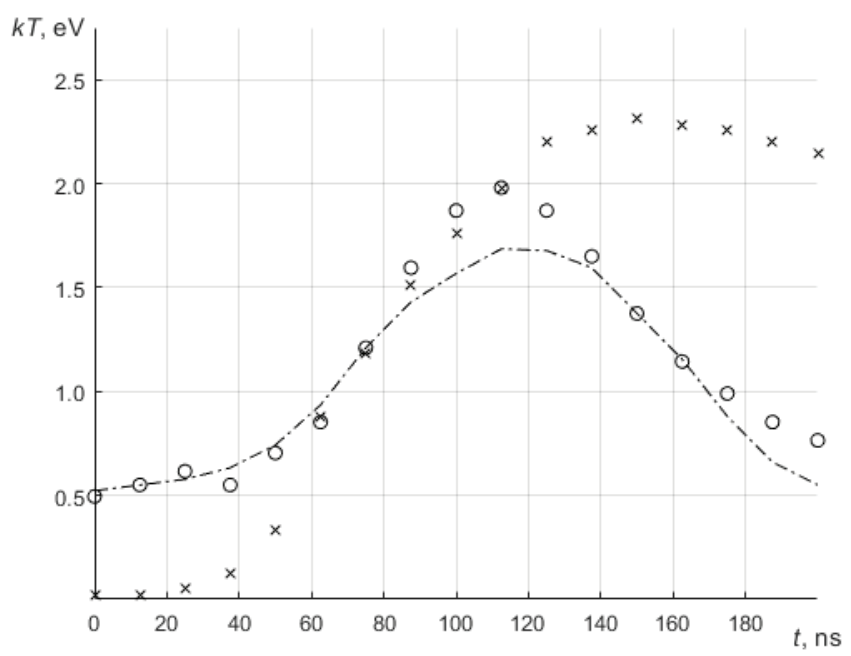

Fig. 4. Comparison of temporal temperature distributions on the interface between the metal vapor and water for the nanosecond explosion [9]. 


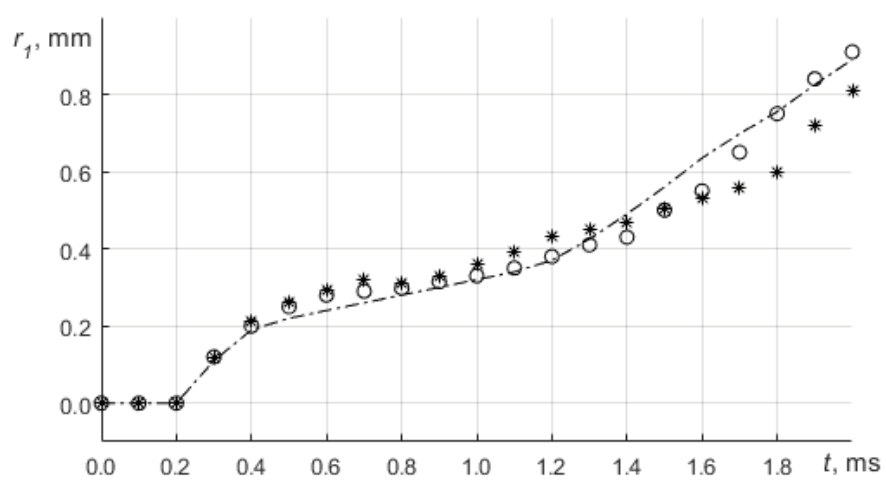

Fig. 5. The dynamics of metal vapor boundary for the microsecond explosion [10].

As the graphs indicate, we have been able to improve the numerical estimation for the thermodynamical evolution of the studied system. This was in part achieved by a careful selection of the auxiliary models, such as equations of state.

\section{Conclusion}

In this paper, we present a mathematical model for a part of the process that results in obtaining materials with programmable properties. The main emphasis is placed on the analysis of various equations of state, allowing calculating sharp changes in the properties of matter in the investigated interval of physical parameters and time intervals. It is obtained that the most rational approach is based on the use of internal or free energy of the thermodynamic system. Test calculations have shown that even simple equations of state give quite satisfactory results.

Further development of the work will go in two directions.

1. Continue testing the mathematical model on more complex equations of state. Ideally, it is desirable to use one or more combined equations that allow to significantly expand the range of the process parameters.

2. Carry out modeling of physical and chemical processes with the use of various metallic conductors as a source of reaction.

\section{References}

1. Pozdeev V A. Applied fluid dynamics of the electric discharge in liquid. (Kiev: Naukova Dumka,1980)

2. Zeldovich, Ya B., and Yu P. Raizer. Physics of shock waves and high-temperature hydrodynamic phenomena. No. FTD-MT-64-514. (Foreign Technology Div WrightPatterson AFB OH, 1965.)

3. Chetverushkin B N. Mathematical modeling of dynamic problems of radiating gas. (Moscow: Nauka, 1985).

4. Naugolnykh K A and Roy N A. Electrical discharges in water.(Moscow: Nauka, 1971)

5. Samarsky A A and Popov Y P. Difference methods for solving problems of gas dynamics. (Moscow: Nauka, 1980)

6. Siegel, R., and C. M. Spuckler. "Approximate solution methods for spectral radiative transfer in high refractive index layers." International journal of heat and mass transfer 37 (1994): 403-413.

7. Kolgatin S N.J.Technical Physics. B 65 (7) 10 (1995) 
8. R. V. Davydov, V. I. Antonov. Simulation of femtosecond pulsed laser ablation of metals. Journal of Physics: Conference Series, Volume 769 (2016), 012060

9. Sasaki, Toru, et al. "Electrical conductivities of aluminum, copper, and tungsten observed by an underwater explosion." Physics of Plasmas 17.8 (2010): 084501.

10. Krasik, Yakov E., et al. "Underwater electrical wire explosion and its applications." IEEE Transactions on Plasma Science 36.2 (2008): 423-434. 